\title{
Quantum Instability of Magnetized Stellar Objects
}

\author{
R. González Felipe ${ }^{1}$, H. J. Mosquera Cuesta ${ }^{2,3}$, A. Pérez Martínez ${ }^{4}$ and H. Pérez \\ Rojas $^{4}$ \\ 1 Departamento de Física and Centro de Física Teórica de Partículas, Instituto Superior \\ Técnico, Av. Rovisco Pais, 1049-001 Lisboa, Portugal \\ 2 Instituto de Cosmologia, Relatividade e Astrofísica (ICRA-BR), Centro Brasileiro de \\ Pesquisas Físicas, Rua Dr. Xavier Sigaud 150, CEP 22290-180, Urca, Rio de Janeiro, RJ, \\ Brazil \\ 3 Centro Latino-Americano de Física, Avenida Wenceslau Braz 71, CEP 22290-140 fundos, \\ Botafogo, Rio de Janeiro, Brazil \\ ${ }^{4}$ Instituto de Cibernética, Matemática y Física, Calle E No. 309, 10400 La Habana, Cuba
}

\begin{abstract}
The equations of state for degenerate electron and neutron gases are studied in the presence of magnetic fields. After including quantum effects to study the structural properties of these systems, it is found that some hypermagnetized stars can be unstable based on the criterium of stability of pressures. Highly magnetized white dwarfs should collapse producing a supernova type Ia, whilst superstrongly magnetized neutron stars cannot stand their own magnetic field and must implode, too. A comparison of our results with a set of the available observational data of some compact stars is also presented, and the agreement between this theory and observations is verified.
\end{abstract}

Key words: Dense matter- equation of state-instabilities-magnetic fields-stars: neutron - white dwarfs

\section{INTRODUCTION}

Many stellar objects are known to be endowed with large magnetic fields (Dryzek et al. 2002, Heyl [1999, 2000). For instance, white dwarfs with surface magnetic fields whose strengths range from $10^{5}$ to $10^{9} \mathrm{G}$ have been discovered (see an earlier set of references in Kemp et al. 1970, Putney 1995, Schmidt \& Smith 1995, Reimers 1996, and the updated list of Suh \& Mathews 2000). Moreover, magnetic field strengths of the order of $10^{20} \mathrm{G}$ have been suggested to exist in the core of neutron stars or pulsars. Recently, in Refs. Chaichian et al.2000 and Pérez et al. 2003] electron and neutron gases in a strong magnetic field were considered with the aim to study the equation of state of white dwarf stars, neutron stars and its relation to supernovae. It was found a pure quantum effect: the appearance of a ferromagnetic configuration in the neutron star interior, which is intrinsically related to the presence of the magnetic field. Such an effect opens the possibility of a quantum magnetic collapse of the gas under consideration. 
This effect is related to the density of the star and its magnetic field, and as such, it allows one to establish a criterium of stability taking into account these physical properties.

The presence of a magnetic field drives the loss of the rotational symmetry of the particle spectrum, which in turn manifests as an anisotropy in the thermodynamic properties of the system. This behavior can be seen through the energy-momentum tensor $\mathcal{T}_{\mu \nu}$, if we recall that the external magnetic field $\mathcal{H}$ induces a magnetization $\mathcal{M}$ in the medium which is described through the relation $\mathcal{H}=B-4 \pi \mathcal{M}(B)$. Here $B$ is the microscopic magnetic field, which is assumed to point along the $x_{3}$ axis in what follows. Starting from the energy-momentum tensor, one can derive the expressions for the pressure components, longitudinal $\left(p_{3}\right)$ and perpendicular $\left(p_{\perp}\right)$ to the magnetic field,

$$
\mathcal{T}_{\perp}=p_{\perp}=-\Omega-B \mathcal{M}, \quad \mathcal{T}_{33}=p_{3}=-\Omega
$$

where $\Omega$ is the thermodynamic potential.

In classical electrodynamics (Landau \& Lifshitz 1970), i.e., when the spin interactions are not taken into account, this anisotropy appears but the results are quite different. Since

$$
p_{\perp}=p_{0}+\frac{B^{2}}{4 \pi}, \quad p_{3}=p_{0}-\frac{B^{2}}{4 \pi},
$$

where $p_{0}$ is the isotropic pressure, one obtains at the classical level

$$
p_{\perp}>p_{3}
$$

This fact explains the oblateness of some astrophysical objects when one studies them in a classical way. Proper examples of this are provided by Shapiro \& Teukolsky 1983 in the case of magnetic white dwarfs, and by Cardall, Prakash \& Lattimer 2001 for the highly magnetized neutron stars. Contrary to these well-known effects, the interplay of magnetic and quantum effects imply that some stars get a cigar-like shape along the $x_{3}$ axis, and some of them may even collapse.

The purpose of this paper is to discuss in more detail and to exploit some of the astrophysical consequences of previous works (Chaichian et al. 2000, Pérez et al. 2003) regarding the appearance of hydrodynamic instabilities in strongly magnetized electron and neutron gases as realizations of the physics taking place inside white dwarfs (WDs) and neutron stars (NSs), respectively. In particular, these instabilities on the configuration of any super critically magnetized stellar object are shown to appear due to the action of quantum-mechanical effects, as the occupation of the particle Landau ground state, which is driven by the cooperative particle spin-magnetic field coupling. These new effects allow us to introduce a new model to show that some WDs may become ultramagnetized and may collapse in a kind of SN type Ia, even without reaching the Chandrasekhar mass limit. As a matter of consistency, we test this theoretical argument in a wide and extensively studied set of astrophysical sources and show that the conclusions drawn from our analysis are in complete agreement with present observations of compact remnant stars, while forbid the existence of some exotic configurations of them.

The structure of the paper is as follows: In Section 2 we present the physics of a magnetized electron gas and apply it to the study of the stability conditions of white dwarfs. Based on this physics, a new model for SN type Ia events is presented, which not necessarily depends upon the WD to reach its critical mass. Section 3 considers the case of a neutron gas, as a model for NSs. We show that the so-called magnetars cannot in principle be formed based on the instability criterium of anisotropic pressures, while canonical pulsars would. Finally, in Section 4 we present our conclusions. 


\section{MAGNETIZED ELECTRON GAS}

In this section we shall study the thermodynamic properties of the degenerate electron gas in very intense magnetic fields and density regimes, which for astrophysical scenarios are the more interesting properties to be investigated. We shall find the conditions that may lead to the vanishing of the transverse pressure of the electron gas, as a model of a white dwarf, which may then undergo a gravitational collapse.

We start by defining the thermodynamic potential as ${ }^{1}$

$$
\Omega=-T \ln \mathcal{Z}
$$

where $T$ is the gas temperature and $\mathcal{Z}$ the partition function. In the case of a magnetized degenerate electron gas, the sum over Landau levels appear in the electron gas configuration and, therefore, the thermodynamical potential can be expressed as

$\Omega_{e}=-\Omega_{0} \sum_{0}^{n_{\mu}} a_{n} B\left[x \sqrt{x^{2}-1-2 n B / B_{c}}-\left(1+2 n B / B_{c}\right) \times \ln \left(\frac{x+\sqrt{x^{2}-1-2 n B / B_{c}}}{\sqrt{1+2 n B / B_{c}}}\right)\right]$,

where we define $\Omega_{0}=e m^{2} /\left(4 \pi^{2} \hbar^{2} c^{2}\right), a_{n}=2-\delta_{0 n}, x=\mu_{e} / m_{e}, B_{c}=m_{e}^{2} /(e \hbar c)$, with $\mu_{e}$ as the chemical potential for electrons and $n_{\mu}$ corresponds to the maximum Landau level for a given Fermi energy and magnetic field strength. The maximum occupancy of these Landau levels is defined as

$$
n_{\mu}=I\left[\frac{B_{c}}{2 B}\left(x^{2}-1\right)\right]
$$

$I[x]$ denotes the integer part of $x$.

The mean density of particles is given by $N_{e}=-\partial \Omega_{e} / \partial \mu_{e}$. In the degenerate limit, i.e., at zero temperature, one obtains

$$
N_{e}=N_{0}\left(\frac{B}{B_{c}}\right) \sum_{0}^{n_{\mu}} a_{n} \sqrt{x^{2}-1-2 n B / B_{c}}
$$

where $N_{0}=m_{e}^{3} /\left(4 \pi^{2} \hbar^{3} c^{3}\right)$. Bearing in mind that the magnetization is defined as $\mathcal{M}_{e}=$ $-\partial \Omega_{e} / \partial B$, from Eq. (5) one obtains

$$
\mathcal{M}_{e}=\mathcal{M}_{0} \sum_{0}^{n_{\mu}} a_{n}\left[x \sqrt{x^{2}-1-2 n B / B_{c}}-\left(x^{2}+4 n B / B_{c}\right) \times \ln \left(\frac{x+\sqrt{x^{2}-1-2 n B / B_{c}}}{\sqrt{1+2 n B / B_{c}}}\right)\right]
$$

where we define $\mathcal{M}_{0}=e m_{e}^{2} /\left(4 \pi^{2} \hbar^{2} c^{2}\right)$. We stress that the magnetization, as well as other thermodynamic quantities, is a function of $B$ and $N_{e}$. Hence, one can write the expression of $\Omega_{e}$ in terms of the magnetization as

$$
\Omega_{e}=-B \mathcal{M}_{e}-\Omega_{0} \sum_{n=1}^{n_{\mu}} n \ln \left(\frac{x+\sqrt{x^{2}-1-2 n B / B_{c}}}{\sqrt{1+2 n B / B_{c}}}\right) .
$$

Since the thermodynamic potential is a measure of the internal pressure in the gas, then it becomes especially interesting to discuss the equation of state of the system at this point. It is worth to note that the latter receives contributions from the partial pressures of the several species involved. By calculating the energy-momentum tensor of this gas (made up of electrically

\footnotetext{
${ }^{1}$ Notice that we are defining the thermodynamic potential per unit volume.
} 
charged particles) one obtains different equations of state for the directions parallel, $p_{3}$, and perpendicular, $p_{\perp}$, to the magnetic field $B$. They read

$$
p_{3}=-\Omega_{e},
$$

and

$$
p_{\perp}=\frac{2 e^{2} B^{2}}{\pi^{2}(\hbar c)} \sum_{n=0}^{n_{\mu}} n \ln \left(\frac{x+\sqrt{x^{2}-1-2 n B / B_{c}}}{\sqrt{1+2 n B / B_{c}}}\right) .
$$

It is readily verified from these expressions that for a positive magnetization the transversal pressure exerted by the charged particles in the presence of a magnetic field is smaller than the longitudinal one by the amount $B \mathcal{M}_{e}$.

There are some situations where densities and magnetic fields are such that only the first Landau level of the particle spectrum is occupied. Eq. (11) shows that when the electrons are confined to the Landau ground state, one can write

$$
p_{\perp}=-\Omega_{e}-B \mathcal{M}_{e}=0,
$$

while higher excited Landau states provide a positive contribution to the pressure. If the dominant contribution to the pressure comes from the electron gas, then the vanishing of $p_{\perp}$ means that the pressure due to the gravitational force, of order $G M^{2} / R^{4}$ ( $R$ is the radius of the star), cannot be compensated, and thus there appears an instability which leads to an abrupt implosion of the electron gas. It is interesting therefore to find the conditions for the occurrence of this confinement to the ground state $(n=0)$ and, consequently, for the triggering of the gravitational collapse of the star.

In general, from Eq. (6) one can write

$$
n_{\mu} \simeq 2 \times 10^{-21} \frac{N_{e}^{2}}{B^{3}},
$$

which essentially defines an instability criterium for all magnetized stars in the plane $\left(N_{e}, B\right)$. In Fig. 1 we show the instability region (dotted area) for a magnetized electron gas in that plane. Any star for which its structural configuration places it inside the dotted region should undergo a transverse collapse due to the vanishing of the pressure transverse (perpendicular) to the magnetic field.

\subsection{White Dwarfs}

Recent observations have pointed out that supernovae type Ia (SNIa), considered as standard candles in the redshift range $0.1 \leq z \leq 1$, seem to provide evidence for our Universe to be accelerating (Riess et al. 1998. Perlmutter et al. 1999), driven by some kind of dark energy: a cosmological constant or quintessence field. However, the premise underlying this suggestion needs to be firmly established on astrophysical foundations. In order to settle out this issue in a conclusive way, the redshift independent nature of SNIa should be demonstrated (Karl et al. 2003 Napiwotzki et al. 2003. Hillebrandt et al. 2003), since only a complete understanding of those events can give the required confidence, if any, to the idea that they are indeed standard candles. In this paper we introduce a new scenario for the SN type Ia events based on the physics presented above, which indicates that another kind of collapse may produce SNIa outbursts.

The leading scenario for SNIa suggests that these explosions stem from the complete disruption of a white dwarf (WD) induced by accretion of mass from a companion star, which forces it to surpass the WD Chandrasekhar mass limit $M_{\text {crit }} \sim 1.445 M_{\odot}$ (Livio 2000). The abrupt conversion of nearly $1 M_{\odot}$ of $\mathrm{C} / \mathrm{O}$ to ${ }^{56} \mathrm{Ni}$ and the ${ }^{56} \mathrm{Ni}$ subsequent decay to ${ }^{56} \mathrm{Fe}$ releases, on 


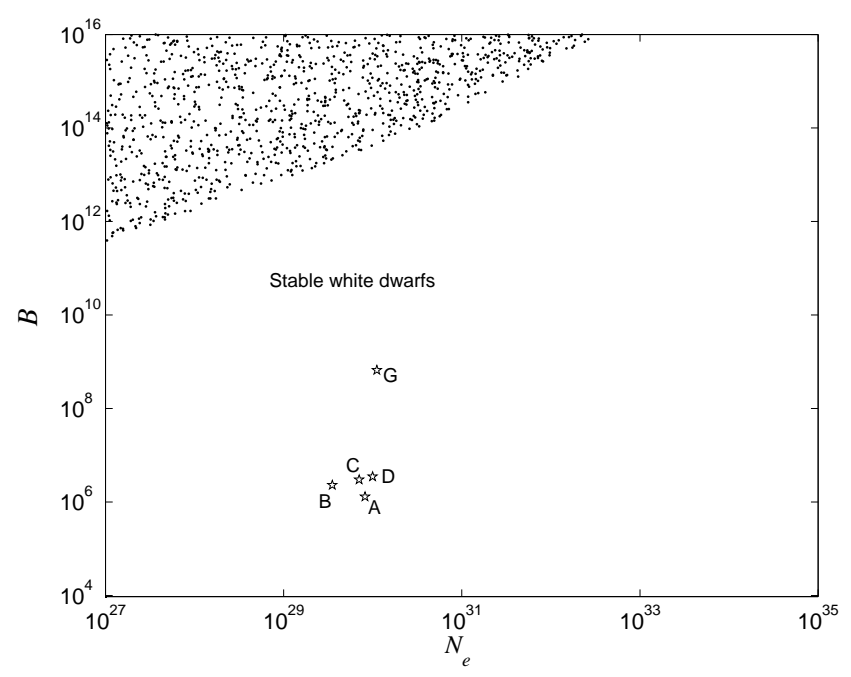

Fig. 1 The instability region (dotted area) in the $\left(N_{e}, B\right)$-plane for a magnetized electron gas. A WD star whose configuration lies inside the dotted region collapses due to the vanishing of the transverse pressure $p_{\perp}=-\Omega_{e}-B \mathcal{M}_{e}$. The labelled points represented by stars correspond to some of the stable white dwarf configurations given in Table 1

\begin{tabular}{cccc}
\hline \hline $\mathrm{WD}$ & $M / M_{\odot}$ & $N_{e} / N_{0}$ & $B$ (in Gauss) \\
\hline $\mathrm{A}$ & 1.28 & 1.4 & $1.3 \times 10^{6}$ \\
$\mathrm{~B}$ & 0.9 & 0.6 & $2.3 \times 10^{6}$ \\
$\mathrm{C}$ & 1.2 & 1.2 & $3 \times 10^{6}$ \\
$\mathrm{D}$ & 1.31 & 1.7 & $3.5 \times 10^{6}$ \\
$\mathrm{E}$ & $0.76-1.0$ & $0.3-0.9$ & $>3 \times 10^{8}$ \\
$\mathrm{~F}$ & $>1.0$ & 0.9 & $3.2 \times 10^{8}$ \\
$\mathrm{G}$ & 1.35 & 1.9 & $6.6 \times 10^{8}$ \\
$\mathrm{H}$ & $?$ & $?$ & $\simeq 10^{9}$ \\
$\mathrm{I}$ & $?$ & $?$ & $\gtrsim 10^{9}$ \\
\hline \hline
\end{tabular}

Table 1 Mass $M$, electron number density $N_{e}$ and surface magnetic field $B$ for some typical magnetic white dwarfs: $\mathrm{A}=\mathrm{PG} 0136+251, \mathrm{~B}=\mathrm{PG} 2329+267, \mathrm{C}=1 \mathrm{RXS}$ $\mathrm{J} 0823.6-2525, \mathrm{D}=\mathrm{PG} 1658+441, \mathrm{E}=\mathrm{LB} 11146 \mathrm{~B}, \mathrm{~F}=\mathrm{Grw}+70^{\circ} 8247, \mathrm{G}=\mathrm{RE}$ J0317-858, H = PG $1031+234, \mathrm{I}=\mathrm{GD} 229$. The above data were taken from Suh \& Mathews 2000 Here $N_{0}=m_{e}^{3} /\left(4 \pi^{2} \hbar^{3} c^{3}\right) \simeq 5.9 \times 10^{29} \mathrm{~cm}^{-3}$ and $M_{\odot} \simeq 1.99 \times 10^{33} \mathrm{~g}$ is the solar mass.

the deserved timescale, an amount of energy just as large as needed to power the observed luminosity and kinematics of a SNIa. In addition, this scenario provides a consistent explanation for the absence of hydrogen in these events. Although this model is well motivated, it has been shown to contain several drawbacks that tend to disfavor it (Hillebrandt et al. 2003).

In an alternative view, recent Hubble Space Telescope observations suggest that a good candidate to SN type Ia progenitors could be coalescing binary WD-WD dubbed as blue strugglers. 
These objects are a class of stars that live in globular clusters orbiting in the outskirts of spiral galaxies. A particular set of these candidates to SNIa are mergers of unequal mass WD-WD binaries that come together because of angular momentum loss via gravitational radiation (GR) emission (Karl et al. 2003, Napiwotzki et al. 2003). The timescale $\left(\sim 10^{6} \mathrm{yr}\right)$ for the system to coalesce is dictated by GR, so that the donor companion has the right time scale to fill its Roche lobe driving then mass transfer onto its heavier orbital partner (Nomoto 1998). However, there exists a debate in the literature whether an explosion does occur under such a dynamics. Some authors contend that view and suggest that more likely a "silent" accretion-induced collapse (AIC) to form a remnant neutron star must take place (Mochkovitch et al. 1997). In overall, the issue seems contentious.

\subsection{Quantum-Magnetic Collapse of a WD as Model of SNIa}

In the lines of the theory presented earlier (Pérez et al. 2003), we suggest here a quite different mechanism in which, for the case of young, rapidly rotating, highly magnetized and massive $\mathrm{C} / \mathrm{O}$ WDs, a direct collapse of a single WD should indeed occur. The overall process is driven by both the quantum and magnetic effects described above.

Rapidly rotating white dwarfs have been studied theoretically (see Shapiro \& Teukolsky 1983 and the extended list of references therein). Despite earlier thoughts dismissing that possibility, based on the fact that the by-the-time observed $\mathrm{H}$ lines in most WD spectra exhibited narrow cores, recent observations, however, confirm that such objects do exist (Karl et al. 2001) among the more than 2400 WDs cataloged up today. The shortest rotation period that has been clearly measured is the $33 \mathrm{~s}$ of AE Aqr, while a good candidate for a slightly faster spin is WZ Sge with a period of $28 \mathrm{~s}$. Nonetheless, there appears to exist a faster class of WDs spinning with periods of $P_{\text {crit }} \sim 27 \mathrm{~s}$, dubbed as DQ Herculis, which, on the other hand, are magnetized WD accreting matter in binaries christened cataclysmic variables.

For non-magnetic white dwarfs, meanwhile, the theoretical view suggests that there exists a critical rotation period (or frequency) of uniformly spinning WDs for which mass shedding at the star equator takes place. The instability sets in for a Keplerian rotation frequency

$$
\Omega_{K}=0.67(\pi G \bar{\rho})^{1 / 2},
$$

where $\bar{\rho}$ is the WD mean density. That relation implies a rotation period $P_{K} \sim 17 \mathrm{~s}$, for stars with mean density in the range $10^{4} \mathrm{~g} / \mathrm{cm}^{3} \leq \rho \leq 10^{7} \mathrm{~g} / \mathrm{cm}^{3}$. However, for WD stars rotating differentially at the relevant temperature the threshold period can be much smaller than the above limit. This is a crucial issue that we will take advantage of in developing our model. According to Shapiro \& Teukolsky 1983 in a differentially rotating WD the ratio between the frequency at the center and at its surface is

$$
\frac{\Omega_{\text {equator }}}{\Omega_{\text {center }}} \leq \frac{1}{5},
$$

for almost all the equilibrium configurations with masses in the range $0.4-0.9 M_{\odot}$. Massive WD models above $1.4 M_{\odot}$ possess surface velocities around $3000-7000 \mathrm{~km} / \mathrm{s}$, which suggests periods around $1 \mathrm{~s}$.

A WD in a binary system may accrete material from its orbital partner in such a way that its angular momentum can rise significantly, and it may rotate differentially. As the material accreted transfers energy and angular momentum, the primary WD star will be spun-up over the time scale

$$
\Delta T_{u}^{s} \simeq \frac{J}{\dot{J}}=\frac{2 M R^{2} \Omega}{5(G M R)^{1 / 2}} \frac{1}{\dot{M}}=4 \times 10^{6} \mathrm{yr} .\left(\frac{M}{M_{\odot}}\right)^{1 / 2}
$$




$$
\times\left(\frac{R}{10^{-2} R_{\odot}}\right)^{3 / 2}\left(\frac{1 \mathrm{~s}}{P}\right)\left(\frac{10^{-8} M_{\odot} \mathrm{y}^{-1}}{\dot{M}}\right),
$$

where $J$ is the angular momentum accreted from the companion, $\dot{J}$ - the applied torque, $P$ - the rotation period, $\dot{M}$ - the accretion rate, and $R$ is the WD radius in units of the solar radius $R_{\odot}$. Notice that a higher accretion rate, as in unequal WD-WD binaries, may reduce the spin-up time scale. Therefore, an essential ingredient of this model is the existence of a WD primary in a binary system which is old enough to having had time to accrete up to the point of being spun-up to its break-up velocity at the equator.

Once we have the star differentially rotating near its break-up period, the conditions are reached for the $\alpha-\Omega$ dynamo mechanism (Duncan \& Thompson 1992, Thompson \& Duncan 1993, 1995, 1996) to start to amplify the WD initial magnetic field. The maximum magnetic field achievable through this process can be as strong as (the equipartition of pressure and kinetic energy)

$$
\frac{B^{2}}{8 \pi}=\frac{M_{\mathrm{WD}} \Omega_{\mathrm{WD}}^{2} R_{\mathrm{WD}}^{2}}{(8 \pi / 3) R_{\mathrm{WD}}^{3}} \longrightarrow B_{c}^{s} \sim 3 \times 10^{14}\left(\frac{P_{i}}{1 \mathrm{~s}}\right)^{-1} \mathrm{G}
$$

with $B_{c}^{s}$ being the supercritical $B$-field and $P_{i}$ the shortest period reached upon undergoing the $\alpha-\Omega$ dynamo phase. This $B_{c}^{s}$ should be generated as the differential rotation is smoothed by growing magnetic stresses inside the WD star. For the typical WD mass, radius and rotation frequency given above, the associated spin energy in the process reads

$$
E_{\Omega i}=\frac{1}{2} M_{\mathrm{WD}} \Omega_{\mathrm{WD}}^{2} R_{\mathrm{WD}}^{2} \sim 10^{51}\left(\frac{P_{i}}{1 \mathrm{~s}}\right)^{-2} \operatorname{erg},
$$

which is similar to the characteristic energy of most SNIa events. This energy plus the gravitational binding energy, which is of the same order of magnitude as the above, would be released during the implosion of the star. The computed amplified magnetic field ${ }^{2}$ plus the matter density of a typical WD will put the star in the instability region showed in Fig 1 according to Eq. (12), where it must undergo a gravitational collapse. Thus, the higher the WD initial $B$ field, the shorter the break-up spin-up time scale. This process may take place well in advance the star reaches the Chandrasekhar critical mass of $1.445 M_{\odot}$.

Since the time scale estimated above is shorter than the age of our universe, certainly old WD stars may accrete enough material so as to rotate at its break-up velocity. At that point its initial $B$-field might be enlarged via the dynamo mechanism, which in turn may drive the star to explode as a consequence of the quantum-magnetic collapse it undergoes. Those systems may be triggering the observed SNIa events.

Whenever the physical properties of the WD star (see Table 1) places it above the instability region depicted in Fig. 1 the WD must implode triggering a supernova-like event with the characteristics of a SNIa. The WD dynamical timescale and the abrupt combustion of its constituent material (electron capture into protons, and rapid process nucleosynthesis to ${ }^{56} \mathrm{Ni}$ ) is to produce the energetics as required on the observational basis. The main reason for this claim is that both characteristics, i.e. the WD rapid spinning and age, can amplify (via the $\alpha-\Omega$ dynamo mechanism) the remnant magnetic field $\left(\sim 10^{8} \mathrm{G}\right)$ up to a critical value to which it may be unstable and thus collapses. In fact, there is strong evidence for young and highly magnetized WDs, as for instance the object RE J0317-858 listed in Table 1 The process we describe is alike to the accretion induced collapse quoted above, but originated from the cooperative action of two different physical effects. At the end, the SNIa explosion must be

\footnotetext{
${ }^{2}$ Notice that the final $B$-field is almost independent of the WD initial $B$-field.
} 
accompanied by a gamma-ray burst of energy $\sim 10^{51} \mathrm{erg}$. This is an interesting signature to look for. A further testable prediction of this picture could be to find no remnant at all after a SNIa! Such a result could be interpreted as if the WD had collapsed into a black hole or a black string, driven by the combination of the quantum and magnetic effects discussed here, and purported in Pérez et al. 2003 .

Were this model, i.e. the collapse of heavy and strongly magnetized WD, realized in nature, it would significantly help to clarify whether SNIa could indeed be considered as legitimate standard candles, since for the collapse to take place in this case the WD mass must be in the range $0.9-1.35 M_{\odot}$, for which the total binding energy released is practically the same $\sim 10^{51} \mathrm{erg}$. Of course, some other observational constraints play some rôle, as for instance the statistics of massive WD-WD binaries, which is relatively small (Karl et al. 2003, Napiwotzki et al. 2003).

In brief, several white dwarfs endowed with strong magnetic fields have been observed and studied (Kemp et al. 1970, Suh \& Mathews 2000, see Table 10. From the analysis of the observational data (Barstow et al. 1995) Greenstein et al. 1982) one can see that our model is in close agreement with those observations. These stars are stable objects. On the other hand, if those WD listed in Table 1 were given an amplified supercritical $\left(B_{u}^{s}\right)$ magnetic field, then they must implode.

\section{MAGNETIZED NEUTRON GAS}

Most of the observed neutron stars are pulsars, i.e., fast rotating neutron stars with strong magnetic fields (Lorimer 1999). They consist mainly of neutron matter with a high central density. As in the case of WDs, a similar study can be performed for neutron stars which are properly described by a degenerate neutron gas (Shapiro \& Teukolsky 1983). In this case, it is straightforward to calculate the thermodynamic quantities starting from the neutron density $N_{n}$. Assuming that the uniform magnetic field $B$ lies along the $x_{3}$-axis, and using the Dirac equation for neutral particles with anomalous magnetic moment (Bagrov \& Gitman 1990) propagating through this field, we get the spectrum as

$$
E_{n}(p, B, \eta)=\sqrt{p_{3}^{2}+\left(\sqrt{p_{\perp}^{2}+m_{n}^{2}}+\eta\left|q_{n}\right| B\right)^{2}},
$$

where $p_{3}, p_{\perp}$ are the momentum components along and perpendicular to $B$, respectively; $m_{n}$ is the neutron mass and $q_{n} \simeq-1.91 \mu_{N}$ is the neutron magnetic moment $\left(\mu_{N}=e /\left(2 m_{p}\right)\right.$ is the nuclear magneton), and $\eta= \pm 1$ are the $\sigma_{3}$ eigenvalues corresponding to the two possible orientations (parallel or antiparallel) of the neutron magnetic moment with respect to the magnetic field.

The thermodynamic properties of a degenerate neutron gas are easily obtained following the same procedure as in Section II and it was the scope of Ref. Pérez et al. 2003, which we revisit now to discuss their astrophysical implications.

Firstly, the neutron density $N_{n}$, thermodynamical potential $\Omega_{n}$, and magnetization $\mathcal{M}_{n}$ read, respectively,

$$
\begin{aligned}
& N_{n}=N_{0} \sum_{\eta=1,-1}\left[\frac{f_{\eta}^{3}}{3}+\frac{\eta y(1+\eta y) f_{\eta}}{2}-\frac{\eta y x^{2}}{2} s_{\eta}\right], \\
& \Omega_{n}=-\Omega_{0} \sum_{\eta=1,-1}\left[\frac{x f_{\eta}^{3}}{12}+\frac{(1+\eta y)(5 \eta y-3) x f_{\eta}}{24}+\frac{(1+\eta y)^{3}(3-\eta y)}{24} L_{\eta}-\frac{\eta y x^{3}}{6} s_{\eta}\right],
\end{aligned}
$$




$$
\mathcal{M}_{n}=-\mathcal{M}_{0} \sum_{\eta=1,-1} \eta\left[\frac{(1-2 \eta y) x f_{\eta}}{6}-\frac{(1+\eta y)^{2}(1-\eta y / 2)}{3} L_{\eta}+\frac{x^{3}}{6} s_{\eta}\right] \text {. }
$$

Here we define the numerical constants: $N_{0}=m_{n}^{3} /\left(4 \pi^{2} \hbar^{3} c^{3}\right) \simeq 2.73 \times 10^{39} \mathrm{~cm}^{-3}, \Omega_{0}=N_{0} m_{n} \simeq$ $4.11 \times 10^{36} \mathrm{erg} \mathrm{cm}{ }^{-3}$ and $\mathcal{M}_{0}=N_{0} q_{n} \simeq 2.63 \times 10^{16} \mathrm{erg} /\left(\mathrm{G} \mathrm{cm}^{3}\right)$. We also set

$$
x=\frac{\mu_{n}}{m_{n}}, \quad y=\frac{B}{B_{n}},
$$

with $B_{n}=m_{n} / q_{n} \simeq 1.56 \times 10^{20} \mathrm{G}$, and introduced the notations:

$$
f_{\eta}=\sqrt{x^{2}-(1+\eta y)^{2}}, \quad s_{\eta}=\frac{\pi}{2}-\arcsin \left(\frac{1+\eta y}{x}\right), \quad L_{\eta}=\ln \left(\frac{x+f_{\eta}}{1+\eta y}\right) .
$$

In the limit $B=0$, Eqs. (20) and (21) reproduce the usual density and thermodynamic potential of a relativistic Fermi gas at zero temperature (Fradkin 1967). We see that the magnetization $\mathcal{M}_{n}$ is a nonlinear function of $B$, and since $\mathcal{M}_{n} \geq 0$ the magnetic response of the neutron gas is ferromagnetic (Pérez et al. 2003).

As we mentioned in the introduction, the key ingredient brought by the anisotropy of pressures is precisely that the magnetization is positive. In fact, one finds that the condition $p_{3}>p_{\perp}$ holds for any study of a highly magnetized Fermi gas. In particular, we remark that for a neutron gas with anomalous magnetic moment this condition is fulfilled.

We also notice that if $x=1+\eta y$, then $f_{\eta}=s_{\eta}=L_{\eta}=0$. For $x<1+y$ the thermodynamic potential $\Omega_{n}$, and consequently, all the thermodynamic quantities in the system become complex. The condition $x=1+y$ defines a curve in the $\left(N_{n}, B\right)$-plane described by the relation

$$
N_{n}=N_{0}\left\{y^{5 / 2}+\frac{5}{3} y^{3 / 2}+\frac{1}{2} y(1+y)^{2}\left[\frac{\pi}{2}-\arcsin \left(\frac{1-y}{1+y}\right)\right]\right\},
$$

which delimits the region where the pressure becomes complex. Since $y \ll 1$ for the magnetic field strengths under consideration $\left(B_{s} \sim 10^{15} \mathrm{G}\right.$ in the NS surface), we can expand Eq. (25) around $y=0$ to obtain the approximate expression

$$
N_{n} \simeq \frac{8}{3} N_{0} y^{3 / 2} .
$$

It is also straightforward to find the curve which corresponds to the vanishing of $p_{\perp}$. Writing $x=1+(1+a) y$, with $a>0$ in Eqs. (20)-(22), and expanding around $y=0$ we obtain to leading order in $y$ :

$$
\begin{gathered}
N_{n} \simeq \frac{2 \sqrt{2}}{3} N_{0} y^{3 / 2}\left[(a+2)^{3 / 2}+a^{3 / 2}\right], \\
p_{\perp} \simeq \frac{2 \sqrt{2}}{15} \Omega_{0} \frac{y^{5 / 2}}{\sqrt{2+a}}\left[2 a^{3}+7 a^{2}+4 a+a^{3 / 2}(5+2 a) \sqrt{2+a}-4\right] .
\end{gathered}
$$

The solution of the equation $p_{\perp}=0$ is given by $a=a_{0} \equiv \frac{3}{5} \sqrt{5}-1 \simeq 0.34$. Substituting this value into Eq. (27) we find

$$
N_{n} \simeq \frac{2 \sqrt{2}}{3} N_{0} y^{3 / 2}\left[\left(\frac{3}{5} \sqrt{5}+1\right)^{3 / 2}+\left(\frac{3}{5} \sqrt{5}-1\right)^{3 / 2}\right] \simeq 3.57 N_{0} y^{3 / 2} .
$$

In Table II we present some results for neutron stars obtained by using other equations of state (EOS), and taking into account that $B$-fields in the core may fulfill the dipolar law, i.e., that $B$ obeys the relation $B=B_{s}\left(R_{s}^{3} / r^{3}\right)$, at least for the dipole component; here $R_{s}$ is 


\begin{tabular}{ccccc}
\hline \hline $\mathrm{NS}$ & EOS & $M / M_{\odot}$ & $N_{n} / N_{0}$ & $B$ (in Gauss) \\
\hline $\mathrm{A}$ & Pol2 & 2.31 & 0.019 & $5.1 \times 10^{20}$ \\
$\mathrm{~B}$ & Pol2 & 2.37 & 0.11 & $1.48 \times 10^{20}$ \\
$\mathrm{C}$ & BJI & 1.96 & 0.18 & $9.1 \times 10^{20}$ \\
$\mathrm{D}$ & BJI & 2.12 & 0.25 & $2.86 \times 10^{21}$ \\
$\mathrm{E}$ & PandN & 1.72 & 0.26 & $5.8 \times 10^{20}$ \\
$\mathrm{~F}$ & PandN & 1.86 & 0.35 & $1.6 \times 10^{21}$ \\
$\mathrm{G}$ & Akmal & 2.22 & 0.21 & $6.4 \times 10^{20}$ \\
$\mathrm{H}$ & Akmal & 2.53 & 0.19 & $3.2 \times 10^{21}$ \\
$\mathrm{I}$ & PCL & 2.09 & 0.054 & $8.7 \times 10^{20}$ \\
$\mathrm{~J}$ & PCL & 1.91 & 0.25 & $1.6 \times 10^{21}$ \\
K & PCLhyp & 2.02 & 0.30 & $6.9 \times 10^{20}$ \\
L & PCLhyp & 1.76 & 0.04 & $1.38 \times 10^{21}$ \\
\hline \hline
\end{tabular}

Table 2 Mass $M$, neutron number density $N_{n}$ and magnetic field $B$ for some neutron star equilibrium configurations computed in Ref. 2001] using different equations of state (EOS). Here $N_{0}=m_{n}^{3} /\left(4 \pi^{2} \hbar^{3} c^{3}\right) \simeq 2.73 \times 10^{39} \mathrm{~cm}^{-3}$.

the star (surface) radius and $r$ is any inner radius of the star. Our results were obtained by using the data from Cardall, Prakash \& Lattimer 2001, where the authors performed "realistic" numerical simulations of strongly magnetized neutron stars. We identify $B_{s}=B_{\text {pole }}$, as of usage in Cardall, Prakash \& Lattimer 2001. Under these conditions, and based on the proposed criterium of stability, we can conclude that those neutron stars would be unstable objects.

In Fig. 2 we plot the curve defined by Eq. (29) in the $\left(N_{n}, B\right)$-plane. For values of $x>$ $1+\left(1+a_{0}\right) y$ the gas pressure is positive and the neutron gas is stable. One can verify, on the other hand, that if $x \leq 1+\left(1+a_{0}\right) y$ the pressure becomes negative or vanishes, thus leading to the transverse collapse of the star.

\subsection{Magnetars}

It has been recently suggested that there might be stellar objects, known as soft gamma repeaters (SGR), whose magnetic field inferred from the spindown of their believed associated pulsars $^{3}$ seems to exceed the critical value: $B_{c}=m_{e}^{2} /(e \hbar c) \simeq 4.41 \times 10^{13} \mathrm{G}$. In particular, it is claimed that these are newly born neutron stars (Mazets et al. 1979, Murakami et al. 1984, Kouveliotou et al. 1999), also called magnetars (Duncan \& Thompson 1992 Thompson \& Duncan 1993, 1995, 1996), with surface magnetic fields as strong as $10^{15}$ G. According to Duncan \& Thompson 1992, Thompson \& Duncan 1993, 1995, 1996, a neutron star with period $P_{i} \sim 1 \mathrm{~ms}$ can support an efficient $\alpha-\Omega$ dynamo. Since neutron stars are formed with significant differential rotation, the associated energy is $E_{\Omega i} \sim 10^{52}\left(P_{i} / 1 \mathrm{~ms}\right)^{-2} \mathrm{erg}$. Thus magnetic fields as strong as $3 \times 10^{17}\left(P_{i} / 1 \mathrm{~ms}\right)^{-1} \mathrm{G}$ can be generated as the differential rotation is smoothed by growing magnetic stresses inside the star. After the available energy is released in the outermost parts of the star, vigorous convection continues to generate much stronger magnetic fields than any previous phase of stellar convection, producing fields larger than the one required to balance the gravitational binding energy density (Duncan \& Thompson 1992, Thompson \& Duncan 1993, 1995, 1996). Thus their result suggests that the $\alpha-\Omega$ dynamo operating in $1 \mathrm{~ms}$ neutron stars might generate surface dipole fields largely stronger than $10^{13} \mathrm{G}$.

\footnotetext{
${ }^{3}$ See also Cuesta 2000 for a different approach.
} 


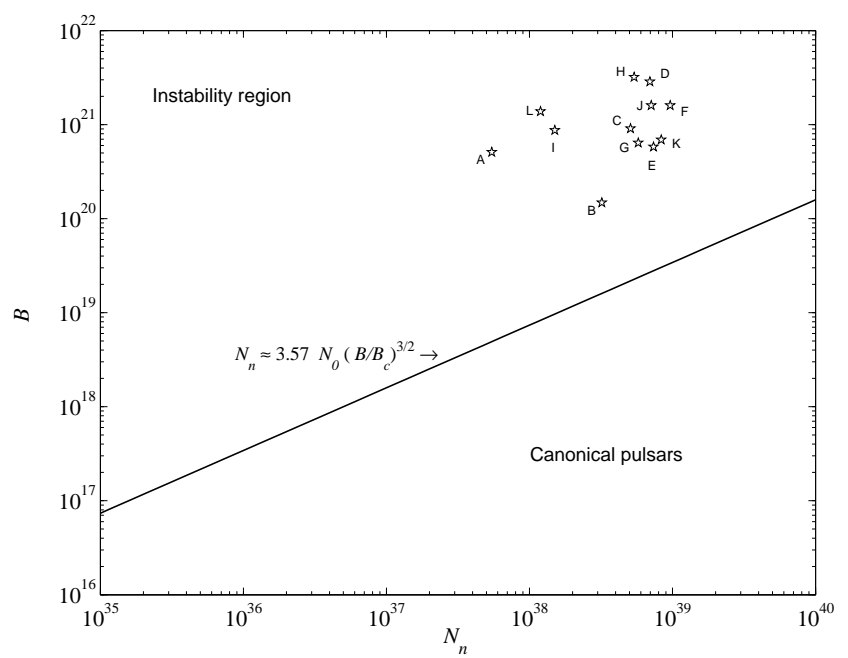

Fig. 2 The instability region in the $\left(N_{n}, B\right)$-plane for a magnetized neutron gas, as a model of a pulsar. A star whose configuration lies above the solid curve defined by Eq. (29) should collapse due to the vanishing of the transverse pressure $p_{\perp}=$ $-\Omega_{n}-B \mathcal{M}_{n}$. The labelled points represented by stars correspond to the neutron star configurations listed in Table 2 and computed using different EOS. The core of any star being trapped in the instability region must implode or collapse as in the quark-nova model (Ouyed et al. 2002).

For this class of compact magnetized stars with $B_{s} \sim 3 \times 10^{17}\left(P_{i} / 1 \mathrm{~ms}\right)^{-1} \mathrm{G}$, our description above predicts that such objects should implode to a magnetically $\left(B \leq B_{c}\right)$ stable configuration, which is not a magnetar! This result, previously obtained in Pérez et al.2003, was later confirmed by Khalilov (Khalilov 2002) and Chakrabarty's group in a very interesting series of papers (Ghosh \& Chakrabarty 2001, Ghosh et al. 2002, Mandal \& Chakrabarty 2002a, 2002b, 2004). In particular, Khalilov in his stability analysis of a degenerate neutron gas in chemical equilibrium with a background of electrons and protons, also included the contribution from the anomalous magnetic moment of the fermions composing the star, as done in Ref. Pérez et al. 2003. We notice, however, that in Ref. Khalilov 2002 a longitudinal collapse is found instead of a transversal one. A detailed discussion of the approach followed in the latter work, which seems to us inappropriate, will be presented elsewhere (Pérez et al. 2005).

As commented above (cf. Fig. 2) some neutron stars described by other EOS producing $B_{s} \sim 3 \times 10^{17} \mathrm{G}$ fall in the region of instability defined by our theory. The attentive reader should note that this magnetic field strength is identical to the one invoked by Kondratyev (Kondratyev 2002) in an attempt to explain the statistical properties of magnetars. By relating intervals of intense activity with sharp, step-like changes of magnetization, due to structural inhomogeneities in the crust and release of its stored magnetic energy, Kondratyev's letter claims that this could be the mechanism triggering SGRs activity. The demagnetization takes place as random jumps associated with magnetic avalanches and sharp energy injection into the magnetosphere develop. Because the nucleons populate discrete energy levels in such a matter, for field strengths $B \sim 10^{16-17} \mathrm{G}$ (and density near $\rho_{\text {nuclear }}$ ), where the energy levels crossover, the nuclei structure changes abruptly to a stepwise field dependence of the nucleus magnetic 
moment on the nucleon magneton. According to this author, the decay of this configuration would then drive the SGRs bursting phases.

However, for a crustal multipolar field configuration one can expect, at the stellar interior, a field strength much higher than $B \sim 10^{17} \mathrm{G}$, at least for the dipole component. This is an idea the astrophysical community is well acquainted with. If this is the case, then our analysis jumps into scene showing (see Fig. 21) that much earlier in the neutron star life it should have collapsed. Notice further that Woods et al. 2002 after studying large torque variations in two SGRs, concluded that within the context of the magnetar model seismic activity cannot account for both the bursts and long-term torque changes unless the seismically active regions are decoupled from one another. The idea is that since the observed changes in spindown rate do not correlate with burst activity then the physical mechanisms behind either phenomena are more likely unrelated. Whether the magnetar model is able to cope with this observational requirement is not apparent.

\section{CONCLUDING REMARKS}

If a hypermagnetized neutron star could somehow be formed in a supernova explosion, the abrupt amplification of its magnetic field will drive it into collapse. Since magnetic flux is dissipated during the implosion via a process analogous to the Sun's coronal mass ejections (as in Malheiro et al. 2004), the most likely outcome of such a collapse may give as a remnant a strange star of canonical magnetic field, black hole or even an exotic black string. Once again, the violent "consumption" of the neutron star nuclear material may trigger the emission of a gamma-ray burst of overall energy $\sim 10^{52} \mathrm{erg}$, inasmuch as in the quark-nova model (Ouyed et al. 2002).

In the case of neutron stars such as the suggested magnetars, the anisotropy of pressures must naturally develop, and the condition for the collapse in the direction perpendicular to the dipole magnetic field could be satisfied (Fig. 2) for the typical values of density and magnetic field strength routinely quoted for these stars.

To summarize, we have presented a consistent theory to discuss the stability of compact remnant stars whose structure is dictated by a combination of quantum and magnetic effects. We have shown that, in general, the theory agrees quite well with current observational data for magnetic WDs (Shapiro \& Teukolsky 1983), canonical pulsars (Lorimer 1999), and the realistic relativistic models of Cardall, Prakash \& Lattimer 2001. A major outcome is the fact that some special configurations of highly magnetized white dwarfs could collapse triggering explosions similar to a SNIa. Besides, some neutron stars endowed with superstrong magnetic fields are shown to be naturally unstable, and therefore should collapse, for their quoted surface magnetic fields. This would drive a powerful supernova explosion followed by a gamma-ray burst, too.

Acknowledgements We are very grateful to João Pulido for stimulating discussions and helpful comments. One of us (A.P.M.) would like to thank the ICTP, IAEA and UNESCO, and CFIF (Lisbon, Portugal) for their financial support and hospitality. The work of R.G.F. has been supported by Fundação para a Ciência e a Tecnologia under the grant SFRH/BPD/1549/2000. H.J.M.C. is a fellow of the Fundação de Amparo à Pesquisa do Estado do Rio de Janeiro (FAPERJ), Brazil, under the contract E-26/151.684/2002, and thanks OEA-ICTP for hospitality in Havana through NET-35.

\section{References}

Bagrov V. G. and Gitman D. M., 1990, Exact Solutions of Relativistic Wave Equations, Kluwer Acad. Publ.

Barstow M. A., Jordan S., O’Donoghue D., Burleigh M. R., Napiwotzki R. and Harrop-Allin M. K., 1995, MNRAS, 277, 971 
Cardall C. Y., Prakash M. and Lattimer J. M., 2001, Astrophys. J., 554, 322

Chaichian M., Masood S., Montonen C., Pérez Martínez A., Pérez Rojas H., 2000, Phys. Rev. Lett., 84,5261

Dryzek J. et al., 2002, Int. Journ. Mod. Phys. D, 11, 417

Duncan R. C. and Thompson C., 1992, Astrophys. J., 392, L9

Fradkin E. S., 1967, Quantum Field Theory and Hydrodynamics, Proceedings of the P. N. Lebedev Institute No. 29, Consultans Bureau

Ghosh T. and Chakrabarty S., 2001, Phys. Rev. D, 63, 043006

Ghosh S., Mandal S. and Chakrabarty S., 2002, astro-ph/0207492

Greenstein J. L. and J. B. Oke, 1982, Astrophys. J., 252, 285

Heyl J., 1999, MNRAS, 306, 333

Heyl J., 2000, MNRAS, 317, 310

Hillebrandt W., Niemeyer J. C., Reinecke M., Travaglio C., 2003, Mem. Soc. Astron. Ital., 74, 942

Karl C. et al., 2001, Rotation velocities of white dwarfs, Astronm. Gessellsch. Abst. Series, 18, S0918, and references therein

Karl C. et al., 2003, Astron. Astrophys., 410, 663

Kemp J. C., Swedlund J. B., Landstreet J. D. and Angel J. R. P., 1970, Astrophys. J., 161, L77

Khalilov V. R., 2002, Phys. Rev. D, 65, 056001

Kondratyev V. N., 2002, Phys. Rev. Lett., 88, 221101

Kouveliotou C. et al., 1999, Nature, 391, 235

Landau L. D., Lifshitz E. M., 1970, Classical Electrodynamics, J. Wiley \& Sons, New York

Livio M., 2000, in The greatest explosions since the Big Bang: Supernovae and Gamma-Ray Bursts, Space Telescope Science Institute

Lorimer D. R., 1999, The neutron star - black hole connection, Proceedings of the NATO Advanced Study Institute, Elounda, Crete, Greece, 7-18 June 1999, Eds. Chryssa Kouveliotou, Joseph Ventura and Ed Van den Heuvel; Dordrecht: Kluwer Academic Publishers, 2001. NATO science series C: Mathematical and physical sciences, 567, p. 3 and 21

Malheiro M., Ray S., Mosquera Cuesta H. J. and Dey J., 2004, astro-ph/0411675

Mandal S. and Chakrabarty S., 2002a, astro-ph/0209462

Mandal S. and Chakrabarty S., 2002b, astro-ph/0209015

Mandal S. and Chakrabarty S., 2004, Int. J. Mod. Phys. D, 13, 1157

Mazets E. P. et al., 1979, Nature, 282, 587

Mochkovitch R., Guerrero J. and Segretain L., 1997, in Thermonuclear Supernovae, Eds. R. RuizLapuente, R. Canal, R. Isern, Kluwer Publishers, 187

Mosquera Cuesta H. J., 2000, Soft gamma-ray repeaters as white dwarf - neutron star relativistic binaries, CBPF preprint NF-080-00

Murakami et al., 1984, Nature, 368, 432

Napiwotzki R. et al., 2003, ESO Msngr, 112, 25

Nomoto K., 1998, Nucleosynthesis in type Ia supernovae and constraints on progenitors, XVIIIth Texas Symposium on Relativistic Astrophysics and Cosmology. Eds. A. V. Olinto, J. Frieman, D. N. Schramm, River Edge, N. J., World Scientific

Ouyed R. et al., 2002, Astron. Astrophys., 390, L390

Pérez Martínez A., Pérez Rojas H. and Mosquera Cuesta H., hep-ph/0011399 See also its extended version: Pérez Martínez A., Pérez Rojas H. and Mosquera Cuesta H., 2003, Eur. Phys. J. C, 29, 111 
Pérez Rojas H., Pérez Martínez A. and Mosquera Cuesta H. J., 2005, Anisotropic pressures in very dense magnetized matter, Rep-ICIMAF/2005

Perlmutter S. et al., 1999, Astrophys. J., 517, 565

Putney A., 1995, Astrophys. J., 451, L67

Reimers D., Jordan S., Koester D., Bade N., Köhler Th. and Wisotzki L., 1996, Astron. Astrophys., 311,572

Riess A. G. et al., 1998, Astron. J., 116, 1009

Schmidt G. D. and Smith P. S., 1995, Astrophys. J., 448, 305

Shapiro S. L. and Teukolsky S. A., 1983, Black Holes, White Dwarfs, and Neutron Stars: The physical of compact objects, John Wiley \& Sons, New York

Suh In-Saeng and Mathews G. J., 2000, Astrophys. J., 530, 949

Thompson C. and Duncan R. C., 1993, Astrophys. J., 408, 194

Thompson C. and Duncan R. C., 1995, MNRAS, 275, 255

Thompson C. and Duncan R. C., 1996, Astrophys. J., 473, 322

Woods P. M. et al., 2002, Astrophys. J., 576, 381

This paper was prepared with the ChJAA LATEX macro v1.0. 